\title{
Concentration and velocity patterns in a horizontal rotating suspension of non-Brownian settling particles
}

\author{
W. R. Matson, ${ }^{1}$ M. Kalyankar, ${ }^{1}$ B. J. Ackerson, ${ }^{1}$ and P. Tong ${ }^{1,2}$ \\ ${ }^{1}$ Department of Physics, Oklahoma State University, Stillwater, Oklahoma 74078, USA \\ ${ }^{2}$ Department of Physics, Hong Kong University of Science and Technology, Clear Water Bay, Kowloon, Hong Kong, China
}

(Received 2 October 2004; published 22 March 2005)

\begin{abstract}
We report a systematic experimental study of concentration and velocity patterns formed in a horizontal rotating cylinder filled completely with a monodisperse suspension of non-Brownian settling particles. The system shows a series of concentration and velocity patterns, or phases, with varying rotation rate and solvent viscosity. Individual phases are studied using both side and cross-sectional imaging to examine the detailed flow structures. The overall phase diagram of the system is mapped out as a function of the rotation rate and solvent viscosity. Attempts are made to analyze the functional form of the phase boundaries in order to understand the transition mechanism between different phases.
\end{abstract}

DOI: 10.1103/PhysRevE.71.031401

PACS number(s): 83.80.Hj, 45.70.Qj, 05.65.+b, 83.10.Pp

\section{INTRODUCTION}

Flows of solid particles suspended in a continuous liquid are ubiquitous in nature and are also encountered in a wide range of technological applications [1-3]. Examples include the flow of mud, glass fibers in polymer solutions, and sedimentation and transport of particles, as well as fluidization phenomena. Many industrial processes in chemical, metallurgical, plastics, pharmaceutical, and food processing involve particulate two-phase flows. Because of their fundamental importance and wide applications, particulate two-phase flows have become an active research area in recent years. Uniform hard-sphere particles suspended in a Newtonian fluid, in which thermodynamic forces and Brownian forces are unimportant, form a model system for the study of particulate flows. Although the single-particle motion is known with high precision, the collective behavior of the particles often shows interesting but unexpected features $[1,4]$. Because of long-ranged hydrodynamic interactions, the particle configuration and structure formation are strongly coupled to the flow field, making the suspension dynamics an interesting and challenging problem in statistical physics and lowReynolds-number hydrodynamics.

Recent experiments [5-9] in a horizontal rotating cylinder or Couette device partially filled with a suspension of either neutrally buoyant or heavy particles revealed that the particles separate into a series of axial bands stacked along the horizontal axis. While generally accepted explanations are yet to be developed, the flowing fluid-air interface is thought to be important to structure formation in particle laden systems. Recently, we carried out an experiment with a settling suspension of uniform non-Brownian particles completely filling a horizontal rotating cylinder [10]. Here there is no surface responsible for pattern formation, yet the system showed a series of sharp pattern changes. Before reaching the centrifugal limit, at which all the particles are spun onto the cylinder wall, the rotating suspension exhibits a series of interesting but unexpected concentration and velocity patterns.

Because of the imbalance between the solvent pressure gradient and the centrifugal force due to rotation, an indi- vidual (heavy) particle should not stay suspended in a rotating cylinder indefinitely. Roberts et al. [11] have shown that a single particle inside a horizontal rotating cylinder will spiral outward continuously until it reaches the cell wall. The traveling time for the particle to reach the cell wall is given by

$$
t_{\text {wall }}=\frac{g}{2 U_{0} \omega^{2}} \ln \left(\frac{R^{2}-x_{0}^{2}}{s^{2}}\right)
$$

where $g$ is the gravitational acceleration, $U_{0}$ is the particle settling velocity (Stokes velocity), $\omega$ is the rotation rate of the cylinder, $R$ is the cylinder radius, and $s$ is the initial displacement from the spiral center $x_{0}$. The value of $x_{0}$ is obtained from the condition $x_{0} \omega=U_{0}$, under which the particle settling velocity $U_{0}$ is balanced by the upward rotation velocity $x_{0} \omega$. For our system with the solvent viscosity $\eta$ $=40$ centipoise $(\mathrm{cP})$ and $\omega=5.241 / \mathrm{s}$, the traveling time $t_{\text {wall }}$ is less than $10 \mathrm{~min}$. Nevertheless, various concentration patterns have been observed in the rotating suspension for several weeks without a noticeable change. The collective behavior of the particles is found to be qualitatively different from the single-particle dynamics.

The rotating suspension is a complex system and has many experimental parameters. They include the rotation rate $\omega$, solvent viscosity $\eta$, particle radius $a$, volume fraction $\phi$, cylinder length $L$, and radius $R$. These parameters give rise to multiple velocity and time scales for the system. Without a theory, it is difficult to know what are the dimensionless control parameters for the system. As a result, one has a huge parameter space to explore experimentally. In fact, several independent experiments [12-15] have been carried out recently in the rotating suspensions. These studies revealed several interesting concentration and flow patterns in the system, but some of them were conducted only in an insolated and rather limited parameter space. Theoretical arguments and calculations [12-16] have been made to explain the observed flow patterns, but they have different assumptions and predictions. A generally accepted theoretical framework for the rotating suspension is yet to obtain. 
(a)

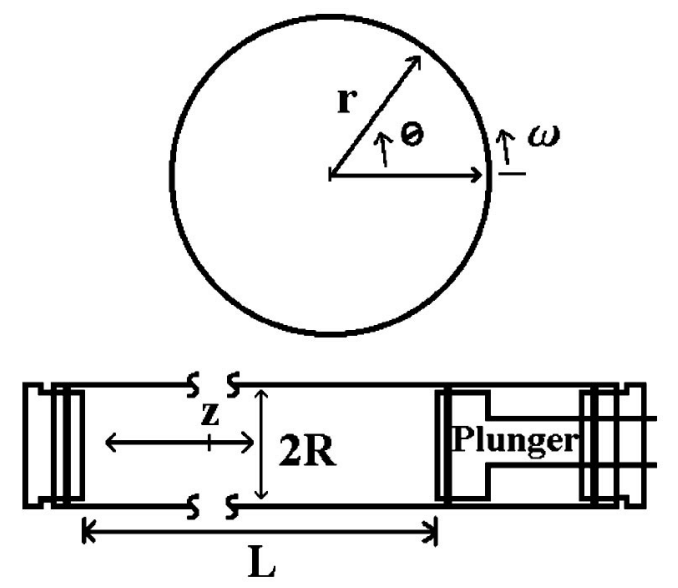

(b)

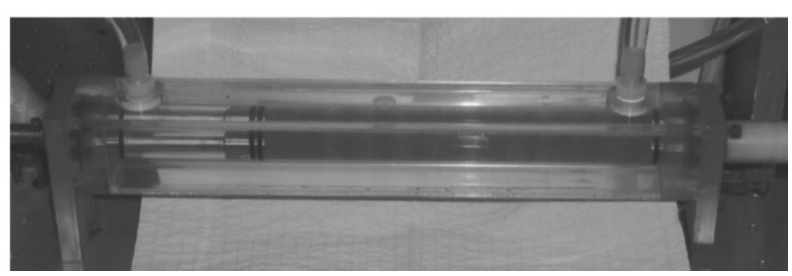

FIG. 1. (a) Construction of the rotating cylinder and the space coordinates used in the presentation of the measurements. (b) The actual experimental setup.

In this paper, we report a systematic experimental study of a settling suspension of uniform non-Brownian particles in a completely filled horizontal rotating cylinder. The overall phase diagram of the system is carefully studied, and the transition boundaries between different phases are mapped out over a wide range of the rotation rate and solvent viscosity. The aim of the paper is to provide the reader an overall picture of the rich dynamics of the system, so that further theoretical analysis can be carried out to give specific guidelines and predictions for the experiment. Such a systematic study is essential to the theoretical understanding of the rotating suspension and will also facilitate further quantitative characterization of the flow structures formed in the system. In the experiment to be presented below, we choose a rotating suspension with fixed values of particle radius $a$, volume fraction $\phi$, and cylinder radius $R$ and study how the concentration and velocity fields of the suspension change with the rotation rate $\omega$ and solvent viscosity $\eta$. The effects of $a, \phi$, and $R$ on the pattern formation in the rotating suspension will be discussed elsewhere $[17,18]$.

The remainder of the paper is organized as follows. We first describe the apparatus and the experimental method in Sec. II. Experimental observations of the concentration and velocity patterns in the rotating suspension are presented in Sec. III. Further discussions of the experimental results are given Sec. IV. Finally, the work is summarized in Sec. V.

\section{EXPERIMENT}

Figure 1 shows the actual experimental setup. The rotating cylinder is made of a Plexiglas tube and is mounted horizontally on a thermally isolated aluminum stand. The inner diameter of the tube is $2 R=1.91 \pm 0.04 \mathrm{~cm}$. Two brass ends are milled to fit the tube and sealed with two o-rings. A sliding plunger is built similarly inside the tube, so that the length $L$ of the tube to be filled in with a solution can be varied. A threaded plug is installed at the center of the plunger to allow air to leak out completely from the tube. Most measurements are carried out with a full tube length $L=22.75 \pm 0.05 \mathrm{~cm}$. The cylinder rotates freely on ball bearings inside a square cooling chamber, which has an inch of clearance around the cylinder. The temperature of the cooling chamber is maintained constant by circulating cold water from a temperature controlled bath and circulator. The temperature stability of the circulator is $0.05{ }^{\circ} \mathrm{C}$, which provides fine control of the solution viscosity. The cooling chamber is made of flat transparent Plexiglas plates to admit the incident light and observe the scattered light by the particles. The use of the flat window eliminates the optical distortions generated by the curvature of the cylinder sidewall and thus improves the quality of visualization of the concentration and velocity fields. The base plate of the aluminum stand is equipped with adjustable legs to facilitate precision leveling of the cylinder and anchor holes to keep the entire system fixed firmly on an optical table.

Figure 1(a) also introduces the space coordinates for the following presentation of measurements. The origin of the coordinate system is chosen to coincide with the center of the rotating cylinder. The $r$ and $\theta$ axes are, respectively, the radial and azimuthal axes in the rotation plane and the $z$ axis is along the axis of symmetry (the rotation axis) of the cylinder. The cylinder is driven by a stepper motor. A thermally insulated coupler is used to prevent motor heat from entering the cylinder system. A microstepping drive controller regulates the motor position with a resolution of $2.5 \times 10^{4}$ steps/ rotation. The controller is stimulated by a homemade indexer, which generates a stable pulse train with 1.0 $\times 10^{-6} \pm 1.0 \times 10^{-9} \mathrm{~s}$ in pulse period. These parameters provide an accuracy of $2.5 \times 10^{-2} \pm 2.5 \times 10^{-5} \mathrm{~s}$ in the rotation period $T$. This fine control of the rotation period (or the rotation rate $\omega=2 \pi / T$ ) allows us to determine the boundary of each dynamic phase accurately.

The stepper motor ratchets the motion of the cell rotation, applying a small-amplitude high-frequency oscillation. The frequency of the oscillation is $\tilde{\omega}=\omega N$, where $\omega$ is the cell rotation rate and $N=2.5 \times 10^{4}$ steps/rotation. For timedependent motion the viscous penetration depth is $\delta$ $=\left[\eta /\left(\rho_{s} \widetilde{\omega}\right)\right]^{1 / 2}$, where $\eta$ and $\rho_{s}$ are, respectively, the viscosity and density of the solvent. For a rotating suspension with $\eta=30 \mathrm{cP}, \quad \rho_{s} \simeq 1.16 \mathrm{~g} / \mathrm{cm}^{3}$, and $\omega=2(1 / \mathrm{s})$, we have $\delta$ $\simeq 22.5 \mu \mathrm{m}$ which is smaller than the size of the particles used in the experiment. Therefore, the small-amplitude highfrequency oscillation produced by the stepper motor should not affect the large-scale concentration and velocity patterns observed in the rotating suspension.

The cylinder is filled completely with an aqueous solution of glycerin with a small amount of liquid detergent $(0.25 \mathrm{vol} \%)$ added to prevent particle aggregation. Two aqueous solutions of glycerin are used to cover the solution viscosity ranged from 8 to $100 \mathrm{cP}$. One aqueous solution has 60 wt \% mass concentration of glycerin and its viscosity var- 
ies from 8 to $22 \mathrm{cP}$ when the temperature of the solution is changed from 30 to $5{ }^{\circ} \mathrm{C}$. The other aqueous solution has 77 wt \% mass concentration of glycerin and its viscosity varies from 19 to $100 \mathrm{cP}$ when the temperature of the solution is changed from 44 to $6{ }^{\circ} \mathrm{C}$ [18]. Measurements of the solvent viscosity at different temperatures are conducted using a Bohlin rheometer.

The particles used in the experiment are uniform glass spheres with an average density $\rho_{p}=2.35 \mathrm{~g} / \mathrm{cm}^{3}$. The particle radius is $a=100 \mu \mathrm{m}$ and the size variation is less than $8 \%$. The volume fraction of the particle suspension is fixed at $\phi$ $=0.023$. The density difference between the particle and the solvent is $\Delta \rho=\rho_{p}-\rho_{s} \simeq 1.19 \mathrm{~g} / \mathrm{cm}^{3}$, and the corresponding Stokes velocity is given by $U_{0}=2 \Delta \rho g a^{2} /(9 \eta)$, where $g$ is the gravitational acceleration. For solutions with viscosity ranged from 8 to $100 \mathrm{cP}$, the corresponding $U_{0}$ varies from 3.25 to $0.26 \mathrm{~mm} / \mathrm{s}$. There are two length scales and two velocity scales in the problem, which give rise to four definitions of the Reynolds number. The Reynolds number based on the particle size and the settling velocity is given by Re $=2 a U_{0} \rho_{s} / \eta$, which varies from $9.4 \times 10^{-2}$ to $7.5 \times 10^{-3}$ in the viscosity range mentioned above. The Reynolds number based on the particle size and wall speed, $\operatorname{Re}=2 a R \omega \rho_{s} / \eta$, ranges from 3 to 0.2 for a maximum rotation rate $\omega$ $\simeq 4 \pi(1 / \mathrm{s})$. The Reynolds number based on the cylinder radius and wall speed, $\operatorname{Re}=R^{2} \omega \rho_{s} / \eta$, ranges from $1.7 \times 10^{2}$ to 13 for a maximum rotation rate $\omega \simeq 4 \pi(1 / \mathrm{s})$. The Reynolds number based on the cylinder radius and the settling velocity is given by $\mathrm{Re}=R U_{0} \rho_{s} / \eta$, which varies from $2.9 \times 10^{-2}$ to 4.5 in the viscosity range mentioned above. The Peclet number given by $\mathrm{Pe}=a U_{0} / D_{0}$, where $D_{0}$ is the Stokes-Einstein diffusion constant for a single particle, ranges from 6.5 $\times 10^{8}$ to $1.2 \times 10^{10}$, implying negligible Brownian motion for all the suspensions used in the experiment.

In the experiment, we use a standard video imaging technique to record the motion and spatial distribution of the particles. A video charge-coupled-device (CCD) camera records particle images in different cross-sectional planes of the cylinder $(r-\theta$ plane $)$ and in the $\theta-z$ planes. To facilitate the imaging in the $r-\theta$ plane (end view), we construct a special short cell $(L=2.25 \pm 0.03 \mathrm{~cm})$ with the same diameter but having a transparent end window for video imaging. The short cell rotates freely on a ball bearing inside a square cooling chamber and is connected to a drive motor outside of the cooling chamber. A sheet of laser light of $1 \mathrm{~mm}$ in thickness is used to illuminate the $r-\theta$ plane of the cell. The whole cell is mounted on a translation stage, so that the flow visualization in the $r-\theta$ plane can be carried out at different locations along the $z$ axis. For the imaging of the long cylinder in the $\theta-z$ plane (side view), back-lit illumination is used. The particle trajectories are visible as streaks in superposed consecutive images. To vary the contrast of the particle images, we use both blue and translucent white glass spheres.

\section{OBSERVATIONS}

Figure 2 shows the "phase diagram" for the rotating suspension as a function of the rotation period $2 \pi / \omega$ and solvent viscosity $\eta$. At low rotation rates, the particles lie and
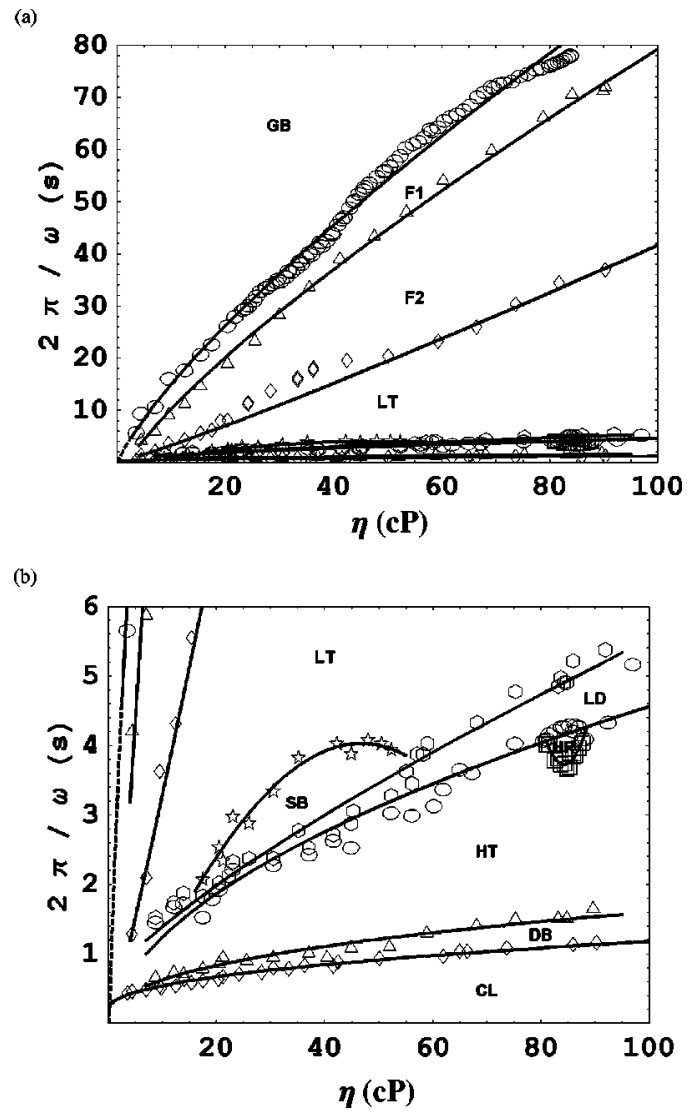

FIG. 2. (a) Overall phase diagram as a function of the rotation period $2 \pi / \omega$ and solvent viscosity $\eta$. (b) An enlarged part of the phase diagram for high-rotation-rate states. The symbols are experimentally determined data points and the solid lines are spline-fitted smooth curves for the phase boundaries. The dashed lines are the extrapolated curves from the fit. GB: granular bed. F1: fingering flow I. F2: fingering flow II. LT: low-rotation-rate transition. SB: stable bands. LD: local-structure dropout. HR: homogenous region. HT: high-rotation-rate transition. DB: discontinuous banding. CL: centrifugal limit.

slide on the bottom of the cylinder, forming a fluidized granular bed. At very high rotation rates, the centrifugal force becomes dominant and all the particles are spun onto the cylinder wall. Between the two extremes, we observe a series of interesting but unexpected concentration and velocity patterns in the rotating suspension. The system exhibits a total of ten different steady states (or dynamic phases) and they are distinguished in the experiment by their unique flow patterns and particle distributions. In this section, we describe each of the phases in order of their appearance with increasing $\omega$. Some of the results have been reported briefly in a rapid communication [10].

\section{A. Granular bed (GB)}

When $\omega$ is zero, the particles lie on the cylinder floor as a loosely packed granular bed. As the cylinder rotates clockwise, the bed is carried up along the rising wall on the left [brighter region in Fig. 3(GB)]. Under gravity the inner layer of particles in contact with the solvent slides downward, cre- 

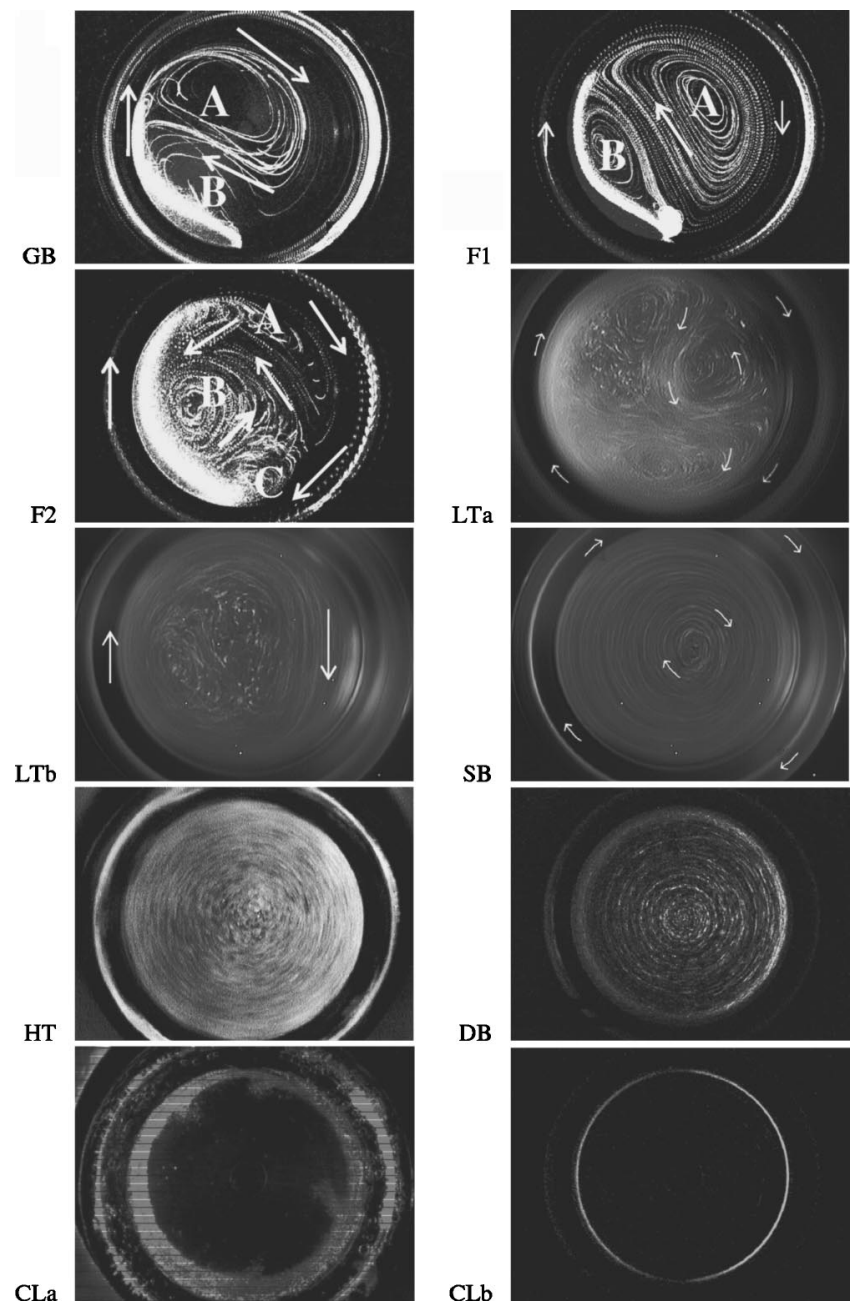

FIG. 3. Cross-sectional view of particle trajectories in the short rotating cylinder. For clarity, 30 sequential images taken at time intervals ranged from $1 \mathrm{~s}$ (for low- $\omega$ sates) to $1 / 30 \mathrm{~s}$ (for high- $\omega$ sates) are superimposed [except (CLa) which is an individual image] and the contrast of the pictures is reversed with white streaks on a black background. The arrows indicate the direction of the local flow and the cylinder rotation. GB: granular bed. F1: fingering flow I. F2: fingering flow II. LTa: low-rotation-rate transition with a remaining granular bed. LTb: low-rotation-rate transition without a granular bed. SB: stable bands. HT: high-rotation-rate transition. DB: discontinuous banding. CLa: centrifugal limit near the transition boundary. CLb: centrifugal limit with all particles on the cylinder wall.

ating a circulation within the bed. A steady state is reached, which results in a fluidized granular flow similar in appearance to that observed for a simple viscous liquid in a partially filled rotating cylinder [19]. This gravity-driven flow produces a counterclockwise rotation for the pure solvent in the central region of the cell. With larger $\omega$, the top leading edge of the bed moves into the upper half of the cell and the particles are injected into and become suspended in the pure solvent region. As shown in Fig. 3(GB), the particle trajectories extend from the leading edge of the granular bed and then bend back to the lower half of the bed due to the counterclockwise flow field of the solvent. It is seen that the par- ticles follow looplike orbits but these orbits are not closed in general.

There are two distinct circulations present in this phase. First, a counterrotating flow (opposite to the cylinder rotation) is located near the granular bed (region $B$ ). Second, the upper part of the bulk fluid (region $A$ ) rotates in the same direction as the cylinder rotation and encompasses the leading edge of the bed, where particles are injected into the solvent. The circulation feeds the injected particles back to the granular bed (region $B$ ).

\section{B. Fingering flow I (F1)}

Fingering flow I is distinguished objectively from the GB phase, when particles in region $A$ opposite the granular bed are no longer carried to the bed. Rather, the counterrotating region near the bed (region $B$ ) and the corotating region opposite the bed (region $A$ ) do not share particles as seen in Fig. 3(F1). These two flow structures are separated by a narrow region, which divides the cross section of the cylinder roughly in half. Particles approaching this region are swept into parallel trajectories on each side of the region, as if a high potential ridge exists along the dividing line. Near the top leading edge of the granular bed [brighter region in Fig. $3(\mathrm{~F} 1)]$, the counterrotating flow meets the wall driven corotating flow, producing a saddle-point-like flow structure. In this region, fluctuations carry some of the particles to the corotating region but most of them stay in the counterrotating structure.

Particles in the F1 phase behave differently from those in the GB phase on a microscopic level. In the GB phase, particles are mostly contained in the granular bed region. Those particles traveling near the upper part of the bed move downward together with the bed with the same velocity and they join the bed downstream in a relatively short period of time. In the F1 phase, however, particles leave the top or leading edge of the bed as a two-dimensional sheet. This is an unstable situation leading to a Rayleigh-Taylor-like instability. Particles clump together into lines or fingers. Initially, two or three particles line up loosely in the flow and then grow into chainlike structures of several millimeters in length and a few particle diameters in width. We call this "finger formation" and hence the resulting flow is named "fingering flow."

Closer examination of the flow field near the leading edge of the granular bed reveals that a finger forms where a selected particle begins to fall and draws one or more neighboring particles along with it. The effective range of the presumed "attractive" hydrodynamic interaction is approximately five particle diameters. As a group of particles, the settling velocity of the fingers is much larger than the Stokes velocity $U_{0}[20]$. As $\omega$ increases, the fingers grow both in number and size, and so does their settling velocity relative the rotating background. The fingers distribute themselves along the $z$ axis uniformly. Figure 4 shows a magnified side view of the fingers formed in the F1 phase. The thin vertical dark strands are the fingers, which fall vertically in the direction of gravity.

Near the low- $\omega$ boundary, the spacing between the fingers is comparable to their own width, only two to three particle 


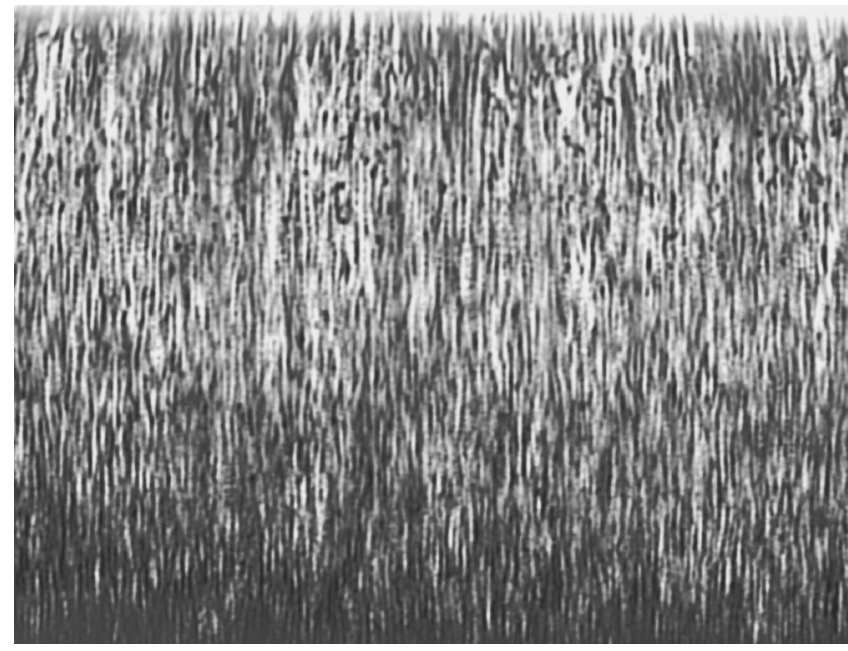

FIG. 4. Side view of chainlike structures (fingers) formed in the F1 phase. For clarity, 30 sequential images are superimposed and the dark streaks show the chainlike structures fall vertically in the direction of gravity. The displayed region is approximately $1.1 \mathrm{~cm}$ by $1.5 \mathrm{~cm}$.

diameter wide. At higher rotation rates, the spacing increases to several millimeters while the fingers themselves remain relatively narrow. Particles within a finger are separated by approximately one to two particle diameters, and they appear to be distributed symmetrically about the central fall line of the finger. Fingers occur somewhat quasiperiodically in terms of the rotation period, giving rise to a time-dependent velocity field. When a finger falls, it follows the granular bed, keeping its radial position $r$ nearly constant until it reaches the base of the bed. Careful particle tracking reveals that the particles shown in Fig. 3(F1) remain within the light sheet during their entire journey, indicating that the fingering flow is essentially two dimensional.

\section{Fingering flow II (F2)}

As mentioned above, the fingers become larger with increasing $\omega$ and thus produce larger disturbances to the flow field in the closed cylinder. Figure $3(\mathrm{~F} 2)$ shows the global flow structure of fingering flow II. The arrows indicate the direction of the local flow and the cylinder rotation. The corotating region $A$ opposite the granular bed [brighter region in Fig. 3(F2)] is pushed further to the upper right corner of the cylinder, and the particles in region $A$ travel a single loop of orbit before returning to the granular bed regions $B$ and $C$. Particles in the counterrotating region $B$ also exhibit closed orbits and can remain suspended for many rotations. Region $C$ near the lower edge of the granular bed is a new circulation, which is absent in Fig. 3(F1). This corotating region is generated by a wall-driven clockwise flow and a faster counterclockwise circulation of the granular bed due the settling of larger fingers. Close observation of the particle-fluid interface separating regions $B$ and $C$ from region $A$ reveals a sharp boundary between the heavily populated region $(B$ and $C$ ) and the nearly void solution $(A)$. Operationally, the F2 transition is defined by the appearance

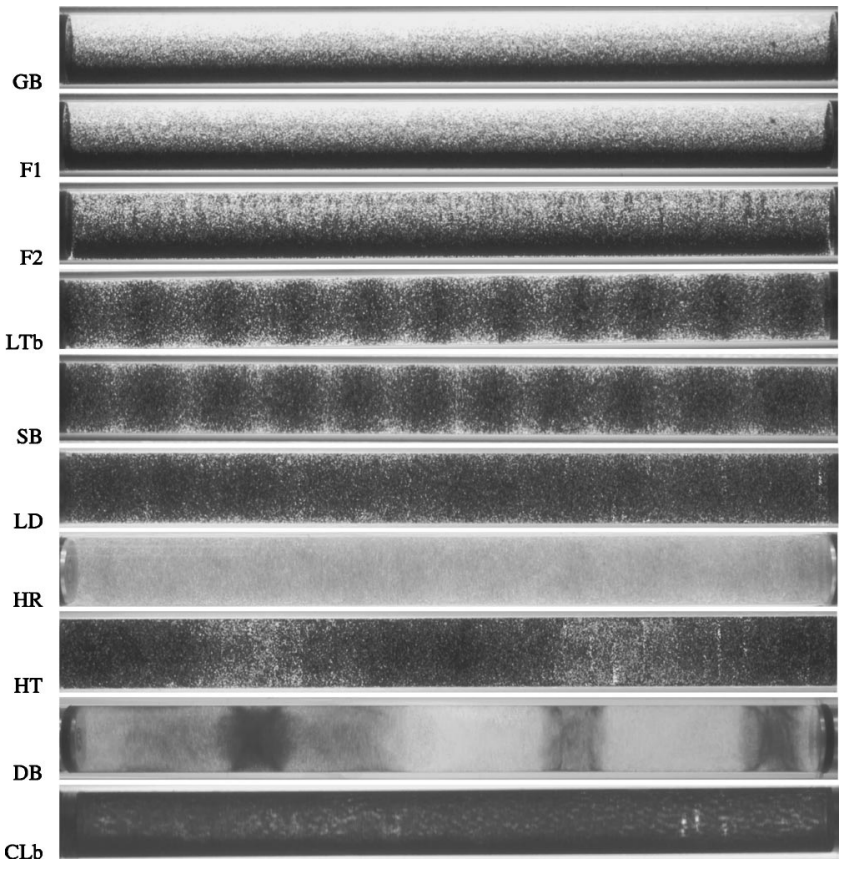

FIG. 5. Side view of the particle distribution in the long rotating cylinder. Darker regions have more particles. GB: granular bed. F1: fingering flow I. F2: fingering flow II. LTb: low-rotation-rate transition without a granular bed. SB: stable bands. LD: local-structure dropout. HR: homogenous region. HT: high-rotation-rate transition. DB: discontinuous banding. CLb: centrifugal limit with all particles on the cylinder wall.

of the circulation $C$ in Fig. 3(F2). The fingers of F1 are no longer absorbed into the granular bed. Rather, the momentum is now so large that inertia carries the fingers beyond the bed and into the corotating flow. This produces the circulation $C$.

As indicated in Fig. 3(F2), fingers start at the top leading edge of the granular bed, where the corotating region $A$ meets with the counterrotating region $B$. They travel along the left side of region $B$ (near the granular bed) until a large portion of the mass is ejected upward between regions $B$ and $C$. When the particles moving inward approach the particlefluid interface, which separates regions $B$ and $C$ from region $A$, they are split almost evenly between regions $B$ and $C$ without disturbing the interface. Similar to the situation in the F1 phase, fingering flow II is also two dimensional.

Figure 5 shows the side view of the particle distribution in the long rotating cylinder. These images are taken using back lighting. Regions with higher particle population absorb and scatter more light and thus exhibit darker color. The fingers in the F2 phase are large enough and become visible in this side view image. As shown in Fig. 5(F2), the thin vertical dark strands on the upper half of the tube are the growing fingers, which are distributed rather uniformly along the $z$ axis. These thin dark strands are invisible in Fig. 5(F1).

Closer examination of the finger detachment near the top leading edge of the granular bed reveals that the fingers in the F1 phase detach from the rising wall at fixed locations of approximately equal distance along the cylinder (side view). In going from the F1 to F2 phases, we find that the detaching 


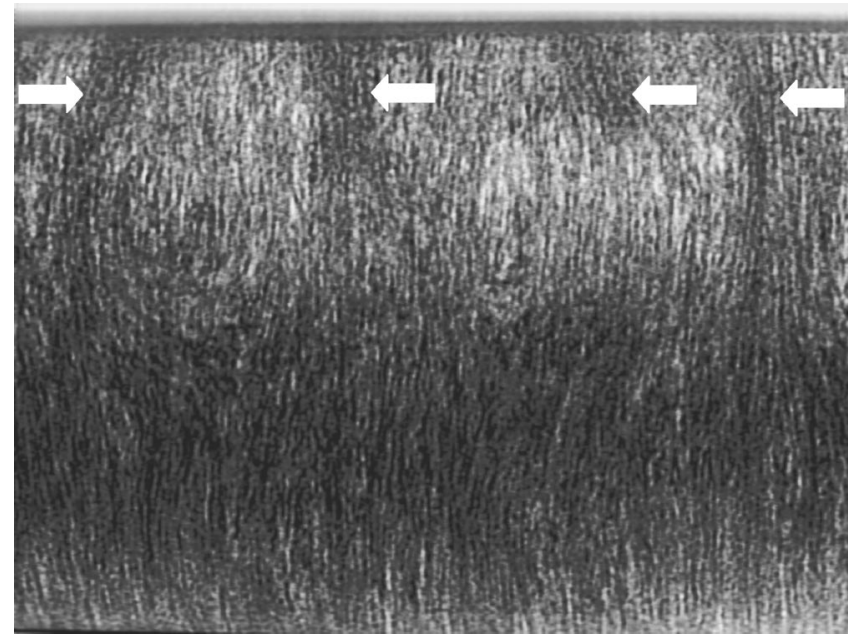

FIG. 6. Side view of fingers formed in the F2 phase. For clarity, 30 sequential images are superimposed and darker regions have more particles. The arrows indicate four fingers visible in the image. The displayed region is approximately $1.9 \mathrm{~cm}$ by $2.5 \mathrm{~cm}$.

position of the settling fingers begins to drift over a small horizontal distance, leading to an apparent zigzaglike path for the falling fingers when viewed at a fixed downstream location as a function of time. Figure 6 shows a side view of the fingers formed in the F2 phase. The arrows point at four fingers visible in the image and they also indicate the drift direction of the detaching position of the settling fingers in the following time period. It is seen that the fingers in the F2 phase have grown much bigger with a typical width of $\sim 2 \mathrm{~mm}$ (10-15 particle diameters).

In a recent experiment, Breu et al. [15] found "traveling waves" in a rotating suspension of $300-\mu \mathrm{m}$-diam glass beads in an aqueous solution of glycerin. Given our results, though in a slightly different region of parameter space, we postulate that the onset of the traveling waves reported by Breu et al. is associated with the transition from the F1 to F2 phases. Because narrow strip images $(512 \times 2$ pixels $)$ were used for flow visualization, only the axial (horizontal) motion of a thin horizontal layer of particles was recorded and the threedimensional structure of the concentration and velocity fields was not studied in the experiment by Breu et al.

\section{Low-rotation-rate transition (LT)}

Operationally, the onset of low-rotation-rate transition is marked by the development of an axial component $\left(v_{z}\right)$ of the finger velocity. This transition is observed in the crosssectional view ( $r-\theta$ plane) by a change in the internal flow structure, as shown in Fig. 3(LTa). In the F2 phase, the motion of the fingers is confined in the $r-\theta$ plane and most fingers fall along a well-defined channel created by the counterrotating flow near the bed. This channel can be seen in Fig. 3(F2). The motion of the fingers becomes three dimensional in the LT phase when the fingers start to fall toward the center of rotation at higher rotation rate. In this case, the radial position $r$ of the settling fingers does not remain constant anymore.
At larger rotation rate, the fingers fall next to the downward moving cylinder wall, as shown in Fig. 3(LTb). Once this occurs, the granular bed is quickly dissolved and all the particles remain suspended. In this case, the fall of the fingers reinforces the lifting of the rising wall on the opposite side of the cylinder, providing a positive feedback to the overall rotating structure. It is seen from Fig. 3(LTb) that the flow field now contains a laminar outer layer following the rotation of the cylinder and an inner fluctuating core region, which, presumably, contributes to the unsteady nature of bandlike structures.

The LT phase has a transition character, which is unique when compared with the other phases discussed above. For the GB, F1, or F2 phases, the basic flow structure does not change much with the rotation rate, as long as the rotation rate stays within the appropriate phase space as shown in Fig. 2. The shape of the granular bed and the general behavior of the fingering flow all remain unchanged throughout the phase region. For the LT phase, however, the basic flow structure changes continuously with increasing $\omega$, as demonstrated by Figs. 3(LTa) and 3(LTb). Near the lower $\omega$ (higher rotation period $T$ ) boundary, the system behaves more like fingering flow II. As the system moves toward the larger $\omega$ (smaller $T$ ) boundary, the fingering flow behavior diminishes and the system resembles more like the stable bands phase, to be discussed in the following section. The bandlike appearance is clearly seen in Fig. 5(LTb). Particles form periodic bands along the $z$ axis with a distinct self-reinforcing flow structure (see Fig. 8 below for more details). The self reinforcing secondary flow is more pronounced at low viscosity than at high viscosity. The bandlike structure is more robust at lower viscosity and fingering is more evident at large viscosity. In this regard, the LT phase resembles a transitional or coexistence phase.

Before reaching the lower $\omega$ boundary of the SB phase, however, these bands are unstable and fluctuate both in time and spatial orientation in a quasiperiodic fashion. These fluctuations are associated with the unstable central core region as shown in Fig. 3(LTb). The fluctuation frequency decreases with increasing $\omega$, from a few seconds at the initial onset of the unstable bands to approximately 1 min near the lower- $\omega$ boundary of the SB phase. The fluctuation frequency also decreases with increasing solvent viscosity, as the viscous drag slows everything down, but the general behavior of the fluctuating bands remain unchanged.

\section{E. Stable bands (SB)}

Secondary flow components parallel to the axis of rotation appear with fingering in the lower $\omega$ boundary of the LT phase. This axial flow continues throughout the phase even as the particle density evidences bands near the larger $\omega$ boundary. What distinguishes this boundary to the stable bands phase is a switching off of the fluctuating velocity components parallel to the rotation axis, while the large-scale band density distribution persists. Once the lower $\omega$ boundary of the SB phase is reached, the self-reinforcing secondary flow pattern associated with the band structure stabilizes and the bands themselves become stationary in space. 


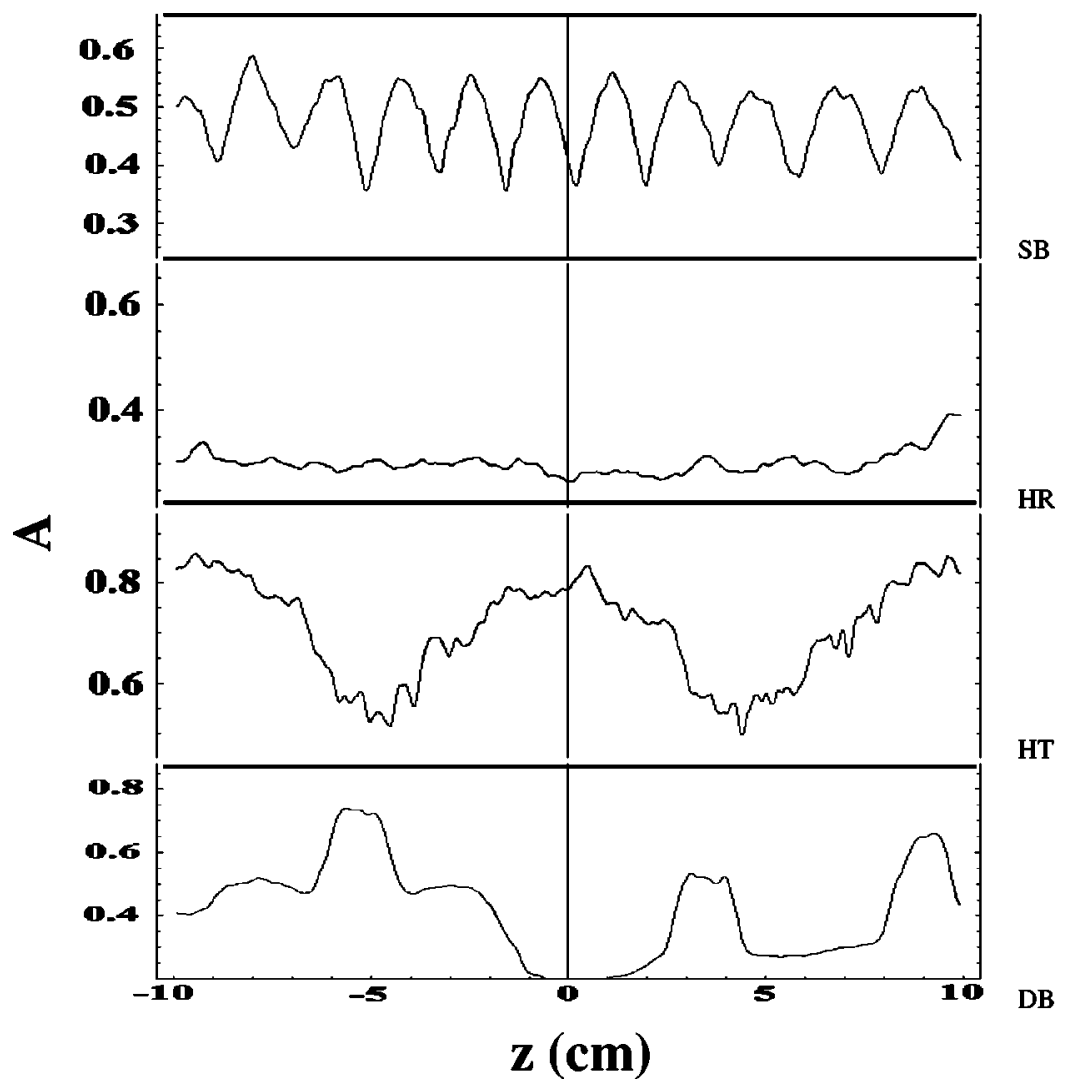

FIG. 7. Measured absorption coefficient $A$ of the transmitted light as a function of the axil position $z$. The transmitted light intensity has been averaged over the cylinder diameter and the vertical line at $z=0$ indicates the middle of the cylinder. The measurements are made in the following phases: SB: stable bands. HR: homogenous region. HT: high-rotation-rate transition. DB: discontinuous banding.

As shown in Fig. 5(SB), the bands are structurally identical and are separated alternately by void regions. The position of the bands remains fixed for a given $\omega$ with fairly uniform spacing, except near the cylinder ends. The average spacing between the bands is $\lambda=2.2 \mathrm{~cm}$, which is $1.2 \pm 0.1$ times the tube diameter. The measurements of the band spacing have been conducted throughout the SB phase space as shown in Fig. 2. Lipson and co-workers [12,13] also reported band formation for nearly neutral buoyant particles in water and other low-viscosity fluids. While their experimental uncertainties are relatively large, the measured band spacing is comparable to our result discussed above. In a different experiment $[17,18]$, we vary the cylinder diameter by a factor of 2 . The particle size is also varied by a factor of 2 and the particle volume fraction $\phi$ is changed from $1.1 \%$ to $2.6 \%$. These measurements reveal that the average band spacing changes only with the cylinder diameter and is independent of other control parameters, such as the solvent viscosity, the rotation rate, and the particle size and concentration.

To characterize the particle concentration variation along the $z$ axis more quantitatively, we convert the twodimensional image files into one-dimensional intensity profiles by averaging out the column pixels in each of the individual images shown in Fig. 5 to obtain the column-averaged transmitted light intensity $\bar{I}(z)$ as a function of the axial position $z$. Because the scattering and absorption of the incident light are proportional to the number of the particles in the optical path (for vanishing $\phi$ ), the measured intensity profile $\bar{I}(z)$ provides information about the particle concentration profile along the $z$ axis. Figure 7 shows the normalized intensity profile of the transmitted light along the $z$ axis. In the plot, the absorption coefficient $A$ is define as

$$
A=\frac{I_{s}-\bar{I}(z)}{I_{s}},
$$

where $I_{s}=255$ is the saturation intensity of the CCD camera used in the experiment. With this definition, darker regions with more particles will have a larger value of $A$. It is seen from Fig. 7 that the particle concentration profile has a sharp triangular wave form (which is also directly visible from a top view). The particle distribution inside each band is symmetric about its central peak position. These maximum concentration planes are stationary in space and the velocities at these locations are vertical and parallel with each other.

As shown in Fig. 3(SB), the particle trajectories in the $r$ - $\theta$ plane become exclusively clockwise in the SB phase. It is seen that the "center" of the particle trajectories does not coincide with the axis of rotation. The trajectory center follows a zigzag path, being closer to the upgoing wall in the $r$ - $\theta$ planes with the highest particle concentration and closest to the downgoing wall at the smallest particle concentrations [as shown in Fig. 3(SB)]. Figure 8 shows a side view of the velocity field inside a stable band. The vertical dashed line $M$ indicates the $r$ - $\theta$ plane with the highest particle concentration. It is seen that the velocity field is symmetric about the maximum concentration line. On each side of line $M$, each particle orbits on its own axis, which tilts at an angle with respect to the rotation axis and points out of the plane of the image toward the less populated band region. The arrows in Fig. 8 indicate the direction of the local flow. Particles are drawn into the dense band region by the secondary flow. Evidently this increased particle concentration produces a larger downward velocity and reinforces the secondary flow. 


\section{F. Local-structure dropout (LD)}

At even larger $\omega$, the secondary circulation shown in Fig. 8 cannot keep up with the increased cylinder rotation and the band structure decays. At first, one segment of the bands disappears and that region appears uniform at lower particle concentration. The other band segments remain unchanged with little or no adjustment in axial location. However, they may absorb particles to increase the required force to maintain the remaining structures. This is shown in Fig. 5(LD). Once the drop-off process starts at a given location, it spreads along the $z$ axis in both directions with increasing $\omega$. It is found that the local-structure drop-off is a reversible process. Reducing the rotation rate reverses the process and restores any destroyed structures in their original positions.

\section{G. Homogeneous region (HR)}

For high viscosity solutions, the drop-off process continues with increasing $\omega$ until all the bands disappear from the entire cylinder. As shown in Fig. 5(HR), the spatial distribution of the particles in the HR phase is fairly uniform throughout the cylinder. The measured transmitted light intensity profile shown in Fig. 7(HR) also reveals this near homogeneous particle distribution across the entire cylinder. The HR phase was referred previously [10] as "rigid-body rotation," because the secondary flows in this phase are small and the whole suspension rotates along with the cylinder. More accurate flow visualization using a synchronized imaging technique with a frame rate exactly the same as the rotation rate reveals that the suspension actually rotates slightly slower than the cylinder rotation. The HR phase is extremely sensitive to the leveling of the rotating cylinder and a slight tilt of less than a tenth of a degree will result in a notable concentration gradient along the tube length. Careful leveling is necessary in order to prevent the gravitationally induced concentration stratification. In the experiment, we are able to keep the cylinder at a perfectly leveled state for several weeks to test the steady-state nature of the HR phase.

It is seen from Fig. 2 that the HR phase occupies a small island region in phase space. The viscosity range is only a few centipoises in width and the largest variation in rotation period is less than $0.5 \mathrm{~s}$ per rotation. Random fluctuations in rotation period or temperature (viscosity) outside this region will destroy the phase completely, and consequently the system cannot reach a true steady state. Therefore, long-term stability for $\omega$ and $\eta$ is needed in the experiment. Even under ideal conditions, it may take up to $18 \mathrm{~h}$ for the system to reach a steady state. The small phase space for the HR phase indicates that a delicate balance among the governing forces is required for this particular flow state.

\section{H. High-rotation-rate transition (HT)}

The high-rotation-rate transition is characterized by largescale concentration variations along the rotation axis ( $z$ axis). As shown in Fig. 5(HT), the particles migrate and form three concentrated (dark) regions extending along the $z$ axis in this case. The dark regions are several centimeters in length and are separated by two less populated (light) regions. This is

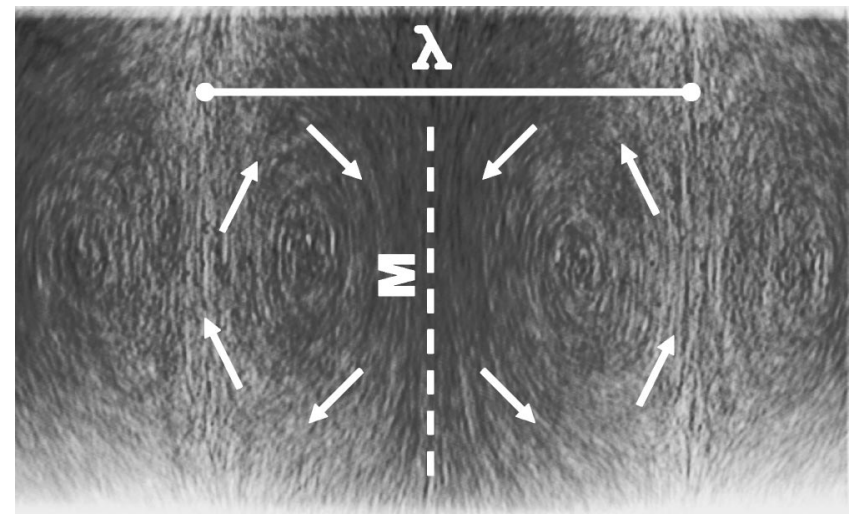

FIG. 8. Side view of the velocity field inside a stable band. For clarity, 30 sequential images taken at $1 / 30 \mathrm{~s}$ intervals are superimposed and darker regions have more particles. The vertical dashed line $M$ indicates the $r-\theta$ plane with the highest particle concentration and the arrows indicate the direction of the local flow. The horizontal solid line $\lambda$ indicates the beginning and ending edges of the single band region.

also shown in the measured transmitted light intensity profile displayed in Fig. 7(HT). Usually we see two or three concentrated regions along the entire length of the cylinder. There is no obvious secondary flow associated with this structure, as in the case of the SB phase and the LD phase. The exact number and the location of the concentrated regions vary with $\omega$ and $\eta$ but their dependence on other experimental parameters, such as the cylinder length $L$, is not known at the moment. From the cross-sectional view shown in Fig. 3(HT), we find that the center of the particle trajectories in the HT phase coincides with the rotation axis.

Similar to the situation in the LT phase, the HT phase also resembles a coexistence phase. Near the lower- $\omega$ boundary, the system resembles the LD phase with a background concentration variation. As the system moves toward the larger$\omega$ boundary, the concentrated regions shrink and the system behaves more like the DB phase, to be discussed in the following section.

\section{Discontinuous banding (DB)}

As $\omega$ increases further, the wide concentrated region in the HT phase contracts to form a very dense region of particles having a width up to one cylinder diameter. The operational definition of the onset of the DB phase is the development of a sharp drop in concentration along the cylinder axis. This is seen when comparing the concentration profiles between Figs. 7(HT) and 7(DB).

Figure 5(DB) shows the concentration profile of the DB phase along the $z$ axis. Hereafter, we refer these dense regions of particles as segregation bands. The location of the band regions appears random and they evidence no interband interaction. From the cross-sectional views we find that each band is composed of a large node of particles located at the rotation center and a layer of particles hovered near the cylinder wall. Between them is a thin layer of sparsely populated region. This is shown in Fig. 3(DB) with more particles accumulated around the center of rotation and a thin layer of 
particles near the cylinder wall [brighter regions in Fig. 3(DB)]. In some cases, the particles are ejected from the central node and slowly spiral outward toward the cylinder wall with a small velocity in the $z$ direction.

High-viscosity suspensions tend to have more but smaller and less-developed segregation bands. These bands have a negligible population of particles near the cylinder wall and a weak but often imbalanced central node. The node tends to oscillate in the $r-\theta$ plane about the axis of rotation and lags behind the cylinder rotation. By comparison, low-viscosity suspensions have fewer but larger and better formed segregation bands. Well-defined segregation bands occur approximately every $8-10 \mathrm{~cm}$, whereas poorly defined bands in high-viscosity suspensions may occur as often as every $2-3 \mathrm{~cm}$. In general, the more distinct the segregation bands are, the greater the separation between the adjacent bands is. It is seen from Fig. 5(DB) that the void region between the bands is completely empty of particles. Unlike the bands in the SB phase, the segregation bands have sharp interfaces with the neighboring unpopulated regions, a discontinuous concentration profile at the interface. This is clearly shown in the measured concentration profile displayed in Fig. 7(DB).

Segregation bands are generally time independent, though some may have mass fluctuations as discussed earlier. For a given rotation rate, the bands form at fixed locations along the $z$ axis. Changing the rotation rate slightly, however, will cause the bands to move axially and reposition themselves. They will again become axially fixed in their new locations, as long as the conditions remain unchanged. The few particles left in the void regions are swept into these band regions, though this process may take much longer time (several hours at $\eta \simeq 40 \mathrm{cP}$ ) compared with that for the segregation structure to form (less than $30 \mathrm{~min}$ at $\eta$ $\simeq 40 \mathrm{cP}$ )

\section{J. Centrifugal limit (CL)}

The large- $\omega$ limit of the phase diagram is the centrifugal limit, at which all the particles spin out to the cylinder wall under the influence of the centrifugal force. This is shown in Fig. 3(CLb), where no particle is found in the interior of the cylinder. Given enough time, all patterns present on the cylinder wall are eliminated and a uniform coating layer of particles is generated on the wall. In our experiment, we typically have an insufficient amount of particles to coat the entire inner wall, which results in one or more single-layered patches of particles on the rotating wall. Figure 5(CLb) shows the side view of the particle coating layer on the cylinder wall.

The transition from the DB phase to the CL phase is one of the sharpest transitions discussed so far. As mentioned above, our resolution in the rotation period is $25 \mathrm{~ms}$ per rotation. The transition between DB and CL takes place within this fine adjustment and is completely reversible. In other words, a change of rotation period of $25 \mathrm{~ms}$ per rotation will cause all the particles to spin out to the cylinder wall. Going back to the previous setting will recreate the segregation bands in exactly the same axial locations, even when the system is allowed to reach a uniform coating on the cylinder

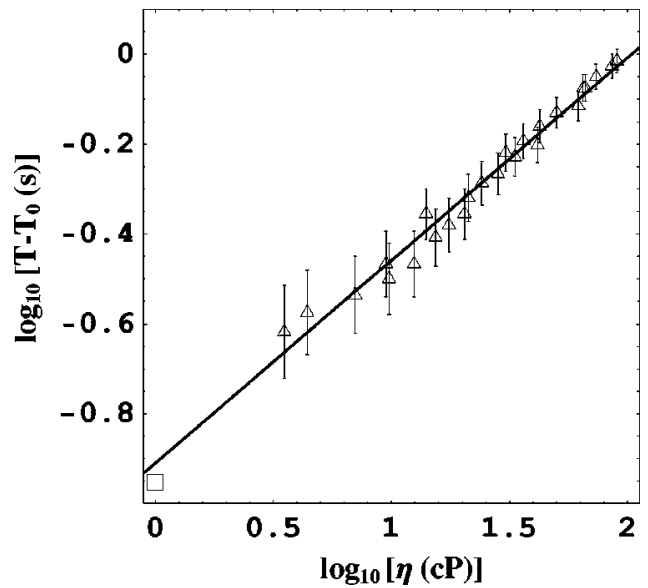

FIG. 9. Measured transition boundary between DB and CL in the $\log _{10}\left(T-T_{0}\right)$ versus $\log _{10} \eta$ plane. The open triangles are obtained in the present study and the open square is extrapolated from the recent experiment by Breu et al. [14]. The solid line shows the power-law fit, $T-T_{0}=0.40 \eta^{0.45}$, to the open triangles.

wall. This reversibility in phase change is of particular interest, since the positioning of the segregation bands has been shown to be $\omega$ dependent.

When reducing $\omega$ toward the boundary between DB and $\mathrm{CL}$, the uniform particle coating layer develops an instability, which causes the particle layer to collapse and form axial chains on the cylinder wall prior to the onset of segregation bands. The axial chains of particles exhibit wavelike motion on the inner surface of the cylinder and they also leave the surface and intrude the interior solvent region. Figure 3(CLa) is an individual image taken in the CL phase near the transition boundary. Four particle chains have intruded the interior solvent region at this particular time. We believe that this instability is of Rayleigh-Taylor type [21] and is a precursor for the onset of segregation bands. Breu et al. [14] also reported the instability of the particle coating layer and the formation of segregation bands in a rotating suspension of $300-\mu \mathrm{m}$-diam glass beads in water $(\eta \simeq 1 \mathrm{cP})$. They found a critical rotation period for the onset of segregation bands with decreasing $\omega$, which agrees well with our result for the transition boundary between DB and CL extrapolated to $\eta$ $=1 \mathrm{cP}$ (see Fig. 9 below). It was suggested [14] that the instability for the particle coating layer is caused by the bouncing of the particles against the cylinder wall.

\section{DISCUSSIONS}

While our discussion on the phase behavior of the system in Sec. III is largely descriptive, the actual determination of each phase is based on the sudden changes of the concentration and flow fields. Information about the particle concentration is obtained by the local particle counting and optical transmission measurements. The flow field is obtained by particle tracking. By watching the motion of the particles over a period of time, one can distinguish these dynamic phases accurately. The velocity and concentration fields are examined both at small length scales comparable to the par- 
ticle diameter and at larger length scales of the order of the cylinder length. Particle tracking is carried out over varying time scales from a second to several minutes.

As described in Sec. III, the dynamic phases are identified according to their unique concentration and velocity patterns. The observed effects are drastic, robust and have been reproduced many times in various cylinders and under different sample conditions. To establish the phase boundary at a given value of $\eta$, we change $\omega$ back and forth with increasingly smaller steps and watch the evolution of the two adjacent phases (or flow states) using various viewing techniques (end and side views). At each step adequate time is given to allow the system to reach a steady state, in which the longtime behavior of the system becomes independent of time. This procedure is repeated at other values of $\eta$, until a complete phase boundary is mapped out in the phase space. The highly accurate control of $\omega$ and $\eta$ produces sharp phase boundaries with no hysteresis.

For example, the transition from DB to CL is so sharp that it lies beyond our resolution limit for $\omega$. It is seen from Fig. 2 that the measured transition boundary between DB and CL has an interesting solvent viscosity dependence. By equating the gravitational acceleration $g$ with the centripetal acceleration $\omega_{0}^{2} R$ at the cell wall, we obtain a characteristic rotation period $T_{0} \equiv 2 \pi / \omega_{0}=0.196$, which is independent of $\eta$ and approximates the phase boundary between DB and CL at the low- $\eta$ limit. To examine the functional form of the transition boundary more closely, we plot the measured transition boundary (open triangles) in the $\log _{10}\left(T-T_{0}\right)$ versus $\log _{10} \eta$ plane, as shown in Fig. 9. It is seen that the measured transition boundary is well described by a power law $T-T_{0}$ $=0.40 \eta^{\epsilon}$ (solid line) with the exponent $\epsilon=0.45 \pm 0.05$. The open square shown in Fig. 9 is obtained from the recent measurement by Breu et al. [14]. Their result also agrees with the power-law fit.

As shown in Fig. 2, different dynamic states occupy separate regions in the phase space, which do not overlap with each other. In addition to these major changes of the flow state, we also find some more subtle structural changes in the concentration and velocity fields, which coexist with the phase changes discussed above. While these secondary structural changes are more gradual in nature, they are an important part of the overall structural changes that occurred in the rotating suspension. Similar to the phase changes discussed above, the secondary structural changes have their own onset conditions with increasing $\omega$. They occur among different dynamic phases and interact with the underlying phases in various degrees. Details about these secondary structural changes are described in Ref. [18].

While we have not been able to verify experimentally a unique set of dimensionless parameters for the system, a simple analysis based on the two-fluid model [22,23] suggests that the rotating suspension has at least three dimensionless parameters. The first parameter is

$$
F_{1}=\frac{\Delta \rho g}{\rho_{s} R \omega^{2}},
$$

which is a ratio of the effective acceleration of gravity for the particles in the suspension to the centripetal acceleration of

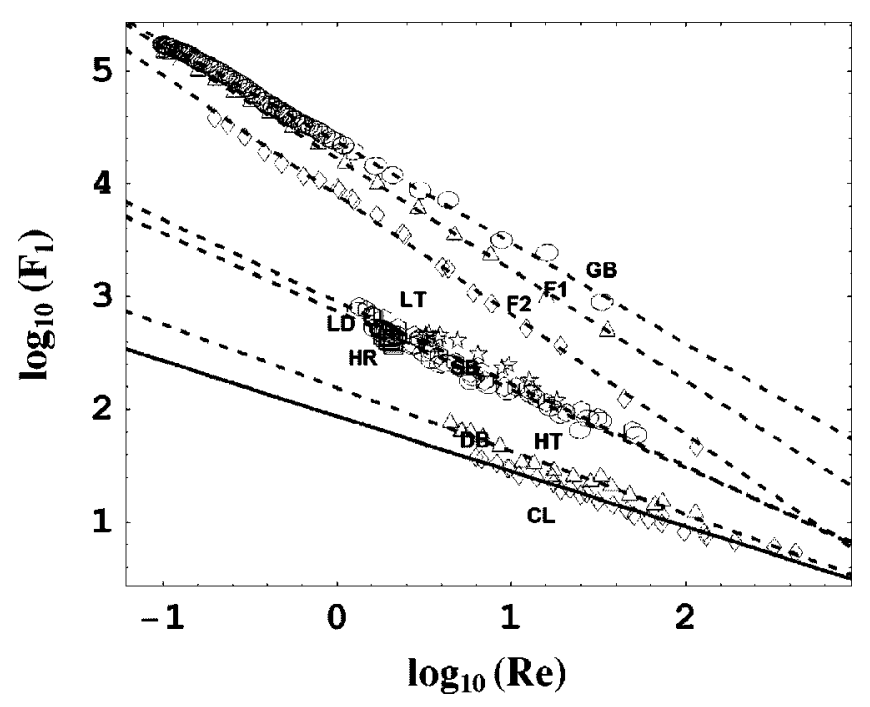

FIG. 10. Replot of the phase diagram shown in Fig. 2 as a function of $\log _{10} F_{1}$ and $\log _{10}$ Re. The symbols show the experimentally determined phase boundaries and the solid line is the powerlaw fit, $F_{1}=87.85 \mathrm{Re}^{-0.49}$, to the diamonds. The dashed lines indicate the linear behavior of each phase boundary in the log-log plot.

the solution at the cylinder wall. The second parameter is the ratio of the Stokes velocity $U_{0}$ to the cylinder wall speed $R \omega$,

$$
F_{2}=\frac{U_{0}}{R \omega}=\frac{2 \Delta \rho g a^{2}}{9 \eta R \omega}
$$

Note that this velocity ratio is proportional to that of the rotation period $2 \pi / \omega$ to the settling time across the cylinder radius $R / U_{0}$. In other words, the settling is balanced by the lift of the upward moving wall. The third parameter is the Reynolds number based on the cylinder radius $R$ and the wall speed $R \omega$,

$$
\operatorname{Re}=\frac{R^{2} \omega \rho_{s}}{\eta} .
$$

Figure 10 is a replot of the phase diagram shown in Fig. 2 as a function of $\log _{10} F_{1}$ and $\log _{10}$ Re. It is seen that the curved phase boundaries in the linear plot shown in Fig. 2 become almost linear, as indicated by the dashed lines in the $\log -\log$ plot shown in Fig. 10. The linear behavior in the log-log plot suggests that the phase boundaries can be described by an effective power law, $F_{1} \sim \mathrm{Re}^{\epsilon}$. For example, the solid line in Fig. 10 shows a power-law fit to the phase boundary between DB and CL. From the fit, we find $\epsilon$ $=0.49$, which is very close to the value obtained in Fig. 9 . The small difference between the two fitted values of the exponent is caused by the fact that an offset constant $T_{0}$ has been subtracted out from the rotation period $T$ used in Fig. 9. It is also seen from Fig. 10 that the measured phase boundaries form three distinct groups. The three-low-rotation-rate boundaries form a group, the two high-rotation-rate boundaries form another group, and the remaining intermediate rotation rate boundaries form a third group. It should be pointed out that the actual variables used in Fig. 10 are $1 / \omega^{2}$ and $\omega / \eta$ (or $\eta / \omega)$. All the other parameters in Eqs. (3) and 
(5) remain constant in the experiment. To verify a unique set of dimensionless parameters experimentally, one needs to change the values of other experimental parameters for the system, such as the particle radius $a$, cylinder radius $R$, volume fraction $\phi$, and density difference $\Delta \rho$. New experiments are underway looking at the influence of these parameters on the phase behavior of the rotating suspension. The results will be reported elsewhere $[17,18]$.

\section{SUMMARY}

A suspension of non-Brownian settling particles in a completely filled horizontal rotating cylinder demonstrates a rich array of concentration and velocity patterns. Individual flow states, or phases, are studied using both side and crosssectional imaging to examine the detailed flow structures. The overall steady-state phase diagram of the system is carefully studied and the transition boundaries between different phases are mapped out over a wide range of the rotation rate and solvent viscosity. While the accurate determination of the phase boundaries is a tedious and lengthy process, such an effort is essential for the understanding of the overall dynamics of the system and the transition mechanism between different phases. Knowing the shape of the phase boundaries and their dependence on the relevant experimental parameters can help us to identify the dominant forces involved and find the correlations between them in forms of dimensionless control parameters.
The experiment suggests that the large number of patterns and rich dynamics found in the rotating suspension come from the interplay among the viscous drag, gravitational force, and centrifugal force. It is likely that the dynamics of the system is controlled by more than one dimensionless parameter. Log-log plots of the transition boundaries over the scaling parameters suggest that the phase diagram of the rotating suspension can be divided into three regions. In the low-rotation-rate regime, hydrodynamic interactions resulting from the settling of individual particles are important. In the high-rotation-rate regime, however, the centrifugal force becomes dominant. The transition boundaries in the intermediate regime show a more complex behavior, suggesting a strong competition among the viscous, gravitational, and centrifugal forces. A further theoretical analysis is needed in order to explain the dynamic behavior and pattern formation of the system in greater detail.

\section{ACKNOWLEDGMENTS}

We thank Anthony J. C. Ladd for useful discussions. The assistance of Mike Lucas and his team in fabricating the experimental apparatus is gratefully acknowledged. This work was supported in part by the National Science Foundation under Grant No. DMR-0071323. P.T. was also supported in part by the Research Grants Council of Hong Kong SAR under Grant No. HKUST603504.
[1] M. C. Roco, Particulate Two-Phase Flow (ButterworthHeinemann, Boston, 1996).

[2] U. Schaflinger, Flow of Particles in Suspensions (SpringerVerlag, Wien, 1996).

[3] C. Crowe et al., Multiphase Flows with Droplets and Particles (CRC, Boca Raton, 1998).

[4] C. Voltz, W. Pesch, and I. Rehberg, Phys. Rev. E 65, 011404 (2001)

[5] O. A. M. Boote and P. J. Thomas, Phys. Fluids 11, 2020 (1999); P. J. Thomas, G. D. Riddell, S. Kooner, and G. P. King, ibid. 13, 2720 (2001).

[6] M. Tirumkudulu et al., Phys. Fluids 11, 507 (1999); M. Tirumkudulu, A. Mileo, and A. Acrivos, ibid. 12, 1615 (2000).

[7] B. D. Timberlake and J. F. Morris, Phys. Fluids 14, 1580 (2002).

[8] N. P. Duong, A. E. Hosoi, and T. Shinbrot, Phys. Rev. Lett. 92, 224502 (2004).

[9] P. Raiskinmaki, J. Astrom, M. Kataja, M. Latva-Kokko, A. Koponen, A. Jsberg, A. Shakib-Manesh, and J. Timonen, Phys. Rev. E 68, 061403 (2003).

[10] W. R. Matson, B. J. Ackerson, and P. Tong, Phys. Rev. E 67,
050301(R) (2003).

[11] G. O. Roberts, D. M. Kornfeld, and W. W. Fowlis, J. Fluid Mech. 229, 555 (1991).

[12] S. G. Lipson, J. Phys.: Condens. Matter 13, 5001 (2001).

[13] G. Seiden, S. G. Lipson, and J. Franklin, Phys. Rev. E 69, 015301(R) (2004).

[14] A. P. J. Breu, C. A. Kruelle, and I. Rehberg, Europhys. Lett. 62, 491 (2003).

[15] A. P. J. Breu, C. A. Kruelle, and I. Rehberg, Eur. Phys. J. E 13, 189 (2004).

[16] J. Lee and A. J. C. Ladd, Phys. Rev. Lett. 89, 104301 (2002).

[17] W. R. Matson et al. (unpublished).

[18] W. R. Matson, Ph.D. thesis, Oklahoma State University, 2004 (available at http://physics.ust.hk/penger/Matson.pdf).

[19] S. T. Thoroddsen and L. Mahadevan, Exp. Fluids 23, 1 (1997).

[20] E. J. Hinch, in Disorder and Mixing, edited by E. Guyon et al. (Kluwer Academic, Dordrecht, 1988), p. 155.

[21] D. H. Sharp, Physica D 12, 3 (1984).

[22] P. R. Nott and J. F. Brady, J. Fluid Mech. 275, 157 (1994).

[23] M. Ungarish, Hydrodynamics of Suspensions (Springer, Berlin, 1993). 